\title{
FIBER-OPTIC BIOSENSORS BASED ON FLUORESCENCE ENERGY TRANSFER
}

\author{
David Meadows and Jerome S. Schultz* \\ Department of Chemical Engineering, University of Michigan, Ann Arbor, MI 48109, U.S.A.
}

(Received 20 May 1987. Revised 1 July 1987. Accepted 19 August 1987)

\begin{abstract}
Summary-A new optical homogeneous biochemical method for the assay of glucose has been developed, based on fluorescence energy transfer between a glucose analog, dextran labeled with fluorescein isothiocyanate (FITC-dextran), and a glucose-receptor protein, Rhodamine-labeled Concanavalin A $(\mathrm{Rh}-\mathrm{ConA})$. When FITC-dextran binds to $\mathrm{Rh}-\mathrm{ConA}$ in solution, and is light-activated, the FITC label transfers its absorbed energy to the Rhodamine label, which then emits light according to its own characteristic fluorescence spectrum. When glucose is added to this solution, the FITC fluorescence intensity increases as FITC-dextran is released from the $\mathrm{Rh}$-ConA and is replaced by glucose. Thus it is possible to determine glucose concentrations directly from the level of FITC fluorescence.
\end{abstract}

One of the key elements in the diagnosis and management of disease is the measurement of (a) specific endogenous substances which either control homeostatic mechanisms or reflect the malfunction of these mechanisms and $(b)$ the blood concentrations of drugs used to remedy the disorders. Most clinical analytical methods are based on techniques which require blood samples to be withdrawn from the patient and taken to laboratories for analysis, and consequently only periodic information is available. Because of the skills needed for their performance, most such analyses cannot be done outside the laboratory, e.g., in the home or workplace.

There are a number of reasons for an intense interest in developing simple, accurate, and rapid clinical assays which can be used by non-analytical chemists, e.g., physicians, nurses, patients. In the intensive care setting, where there is a high level of expertise, there is a need for continuous monitoring of blood for metabolites (e.g., glucose) and relatively toxic drugs (e.g., methotrexate, gentamicin). There is also an increasing need for patient self-monitoring, such as blood glucose by diabetics, and at the intermediate level, clinical analysis to be performed in the doctor's office to help in rapid diagnosis.

Until very recently, most approaches for miniature continuous biosensors have been based on electrochemical detectors. These can be broadly classified as potentiometric or amperometric devices. ${ }^{1.2}$ The usual electrochemical biosensor consists of a "biochemical" layer containing enzymes, organelles, or cells which are held in place in front of an electrode by a dialysis membrane. The literature is extensive, the groups of Rechnitz ${ }^{3}$ and Suzuki $^{4}$ being particularly prolific.

*To whom correspondence should be addressed.
Recently, increasing attention is being given to miniaturization of these electrochemical sensors by use of thin-film integrated circuit technology. $5-7$

There are several problems with electrochemical sensors that have not yet been overcome, however.

1. For potentiometric detectors there is usually persistent drift of the working-cell potential with time, resulting in a need for frequent standardization with solutions of known analyte concentration.

2. The effect of consumption of analyte in the measurement is aggravated for amperometric devices because of the limitations in diffusional transport between the transducer compartment and the detector element.

Thus, enzyme electrodes have had only a "minor impact on medical measurement" ${ }^{8}$ because the use of electrochemical biosensors has mainly been in off-line systems where samples can be alternated with standards.

A primary focus of our research on biosensors has been to utilize an optical analytical method that will be an alternative to the electrochemical biosensor for on-line concentration measurements. There are some inherent advantages to use of spectrophotometry, fluorimetry and light-scattering for analytical purposes, because of the higher information content of the optical domain compared to electrochemical phenomena. Thus spectral features such as absorption or fluorescence at selective frequencies, provide additional degrees of freedom to characterize and quantify a given analyte in a mixture with other substances.

By use of optical-fiber waveguides, spectrophotometric methods can be miniaturized to monitor very small samples, since the active portion of the sensor is about $1 \mu 1$ in volume. This capability has 


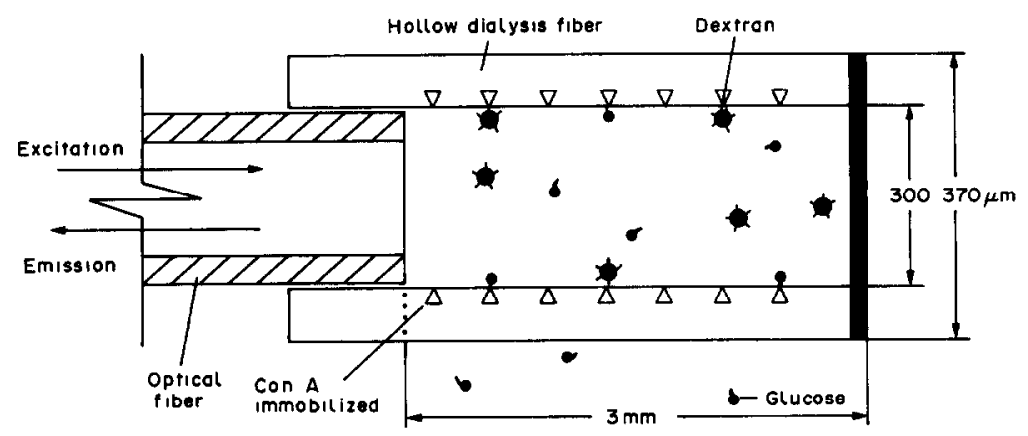

Fig. 1. Schematic of heterogeneous affinity sensor transducer element.

become especially significant in recent years owing to the ready availability of a variety of optical fibers and optoelectronic devices for light-sources and detectors. ${ }^{9}$ Optical fibers have been used to fabricate micro-colorimeters and micro-fluorimeters, ${ }^{10}$ but it is only recently that optical-fiber detector elements have been coupled to biochemical reactions for the purpose of developing miniaturized biosensors. ${ }^{11}$

The required selectivity was conferred on the biosensor by using a selective bioreceptor (Concanavalin A) and competitive binding between an analoganalyte (FITC-dextran) and the analyte (glucose). ${ }^{12}$ A schematic diagram of the optical biosensor is shown in Fig. 1, along with typical dimensions of the active transducer element. In this sensor, the receptor is immobilized on the inner wall of the hollow dialysis fiber. Some typical calibration curves with different analytes are shown in Fig. 2. The response of the sensor displays the expected pattern for a competitive assay, namely a more or less linear response at low concentration and a leveling off at higher concentrations as the competing analyte is displaced from receptor sites. In addition, the different curves demonstrate that the sensitivity of the sensor is related to

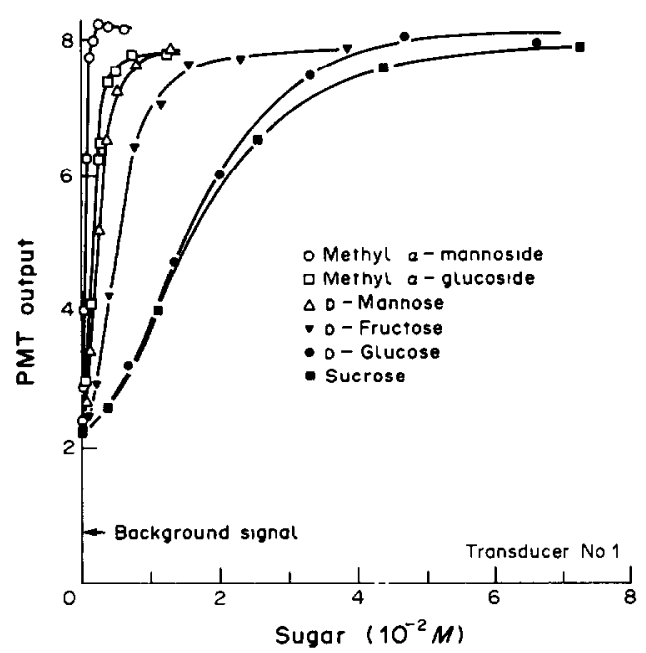

Fig. 2. Effect of binding constant on sensor response. the equilibrium binding constant between analyte and receptor; the higher the binding constant, the greater the sensitivity.

A major concern in the performance of any sensor is its rate of response to changes in concentration. There are three physical/chemical processes that contribute to the dynamic behavior of this sensor: $(a)$ diffusion of the analyte through the wall of the hollow fiber, $(b)$ the competitive displacement reaction between analyte, competing analyte and receptor sites, and $(c)$ diffusion of the various species within the hollow-fiber compartment. The time constants for $90 \%$ completion of these individual steps have been estimated or measured ${ }^{12}$ and found to be about $5 \mathrm{~min}$ for glucose diffusion through the wall of the dialysis fiber, about $1 \mathrm{sec}$ for the displacement reaction (at the concentration conditions within the lumen), and about $3 \mathrm{~min}$ for the diffusion of components within the lumen. The overall time constant for various fabricated sensors ranged from 6 to $12 \mathrm{~min}$. The analyses indicated that faster response could be achieved by making the sensors with hollow fibers with a smaller lumen diameter and/or thinner walls.

The Schultz and Mansouri sensor gave very good short-term stability during in vivo glucose monitoring in a heparinized dog, and good long-term stability during in vitro experiments. However, further testing of the sensor raised several pertinent questions about long-term in vitro stability. In our most recent work, we have been trying to improve on the design.

One major simplification of the sensor is to eliminate the need for chemical immobilization of the bioreceptor (ConA) on the hollow fiber wall to achieve spatial separation of the free and bound forms of FITC-dextran. The chemical immobilization has often been difficult to reproduce and the alignment of the optical and dialysis fibers must also be carefully maintained. If the chemical immobilization step could be eliminated, physical degradation of the dialysis fiber would no longer be a factor. Also, the Schultz and Mansouri sensor is not capable of automatic on-line recalibration to correct for drift of the optical and electrical components. Any shift in the calibration must be corrected by 
using a series of standard glucose solutions. We believe that a new optical homogeneous assay which we have recently developed for glucose may be used in the design of an improved sensor.

The new assay has all the reaction species in one phase and is based on a fluorescence energy transfer technique. Most such methods have been developed for haptens bearing a chromophore that can quench the intrinsic ultraviolet fluorescence of antibody tryptophan residues ${ }^{13-15}$ as the hapten and antibody bind together. However, the technique has received relatively little attention for fluorophores that absorb in the visible region. ${ }^{16,17}$ Our approach employs two labels that absorb and fluoresce in the visible region, FITC-dextran as a donor (fluorescer) and Rhodamine-labeled $\operatorname{Con} A(R h-C o n A)$ as the acceptor (quencher). The competing reactions for the system are given in equations (1) and (2).

Glucose + Rh-ConA $\rightleftharpoons$ Glucose $: \mathbf{R h}-$ ConA (Analyte) (Receptor) $\rightleftharpoons$ (Analyte: Receptor)

FITC-dextran + Rh-ConA

(Competing Analyte) (Receptor)

$$
F_{(\text {Competing Analyte: Receptor })}^{\text {FITC-dextran: } \mathrm{Rh}-\text { ConA }}
$$

The efficiency of the energy transfer process is directly related to the overlap of the emission spectrum of the donor with the absorption spectrum of the acceptor. The hatched region in Fig. 3 shows that there is relatively good spectral overlap of the fluorescein emission spectrum, maximum at $520 \mathrm{~nm}$, with the Rhodamine absorption spectrum, maximum

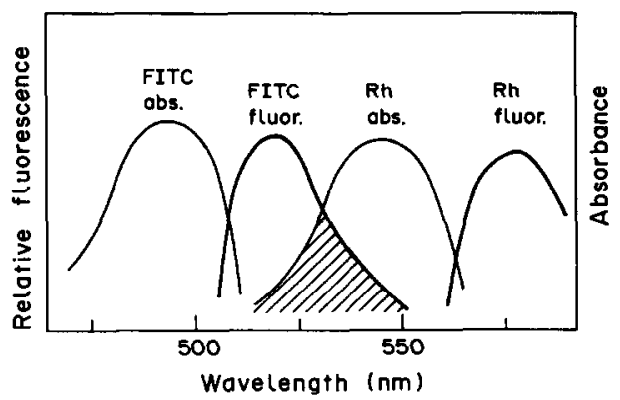

Fig. 3. Overlap of FITC emission spectrum with Rhodamine absorption spectrum.

at $550 \mathrm{~nm}$. Increasing the overlap of the spectra should increase the efficiency of resonance energy transfer between the chromophore pair. When FITC-dextran reversibly binds to $\mathrm{Rh}-\mathrm{ConA}$, the two chromophores are close enough together $(<50 \AA)$ for a significant portion of the fluorescein signal to be quenched by the Rhodamine. As glucose is added to the system, FITC-dextran is liberated from the $\mathrm{Rh}-$ ConA, causing the fluorescein signal to increase. With this system, it is possible to measure a wide range of glucose concentrations by adjusting the concentrations of each component to give the highest sensitivity over the desired glucose range. An added feature of the system is that the Rhodamine fluorescence signal is not significantly affected by the energy transfer, so it can be used as the internal reference for calibration purposes. Because the assay is homogeneous, it is possible to eliminate all immobilization steps and to scale down the assay from the $1-10 \mathrm{ml}$ bench scale to the $1-\mu 1$ sensor level. Figure

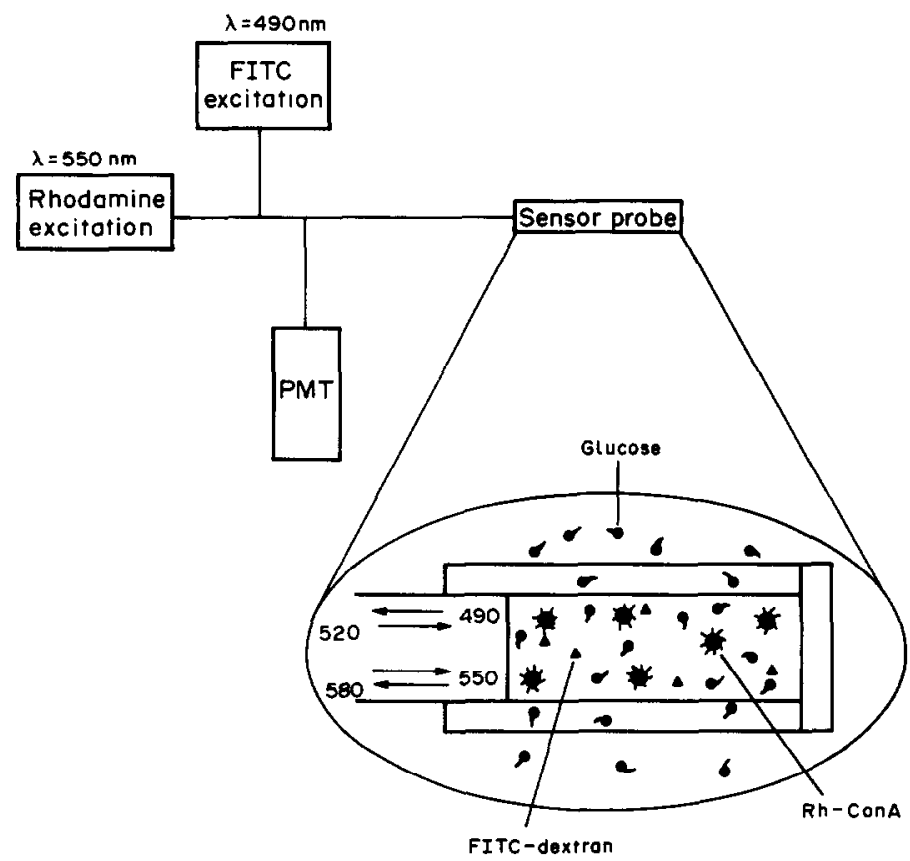

Fig. 4. Schematic of affinity sensor transducer element modified for homogeneous assay. 
4 shows how the sensor would be constructed. Light at two different wavelengths, 490 and $550 \mathrm{~nm}$, would be alternately passed into the fiber, and the resulting signal recorded. A change in the Rhodamine signal would indicate a drift in the light-source or detector, and a change in the FITC signal would indicate a change in the glucose concentration. This flexibility will make it easy to develop sensors for many other low molecular weight $(<1000)$ analyte systems by utilizing a selection of commercially available antibodies, $\mathrm{F}_{\mathrm{AB}}$ fragments, binding proteins, etc.

\section{EXPERIMENTAL}

All fluorescence measurements were made with a specially designed spectrofluorimeter operated in the ratio mode. For measuring fluorescein quenching during energy transfer experiments, the excitation and emission wavelengths were 470 and $520 \mathrm{~nm}$, respectively. All experiments were performed at $25^{\circ}$ in $0.05 \mathrm{M}$ phosphate buffer (containing $0.15 \mathrm{M}$ sodium chloride) at $\mathrm{pH} \mathrm{7.4}$. For the quenching curves, a sample containing the desired FITC-dextran concentration was added to a cuvette in the spectrofluorimeter. The fluorescence intensity of the fluorescein was recorded at a single wavelength, $520 \mathrm{~nm}$, after each $\mathrm{Rh}-\mathrm{ConA}$ addition. Additions were made without removing the cuvette from the holder, and corrections were made for dilution and the inner filter effect from Rhodamine. Glucose titrations were performed similarly, except that the initial solutions contained both FITC-dextran and Rh-ConA at the desired levels. There was approximately a 5-min delay between each addition of glucose of $\mathrm{Rh}$-ConA to ensure that the binding reaction had reached equilibrium.

FITC-labeled dextran, MW $7.0 \times 10^{4}$, was obtained from Pharmacia (Piscataway, NJ), and Rh-ConA from Sigma (St. Louis, MO), U.S. Biochemicals (Cleveland, $\mathrm{OH}$ ) and Polysciences (Warrington, PA).

\section{RESULTS}

Energy transfer between fluorescein and Rhodamine was demonstrated by stepwise addition of a $\mathrm{pH}-7.4$ buffered solution of $\mathrm{Rh}-\mathrm{ConA}$ to a solution of the FITC-dextran. Figure 5 shows that the fluorescein signal significantly decreased as the $\mathbf{R h}-$ ConA concentration was increased. As much as $45 \%$ of the fluorescence was quenched at an $\mathrm{Rh}-$ ConA concentration of $0.33 \mathrm{mg} / \mathrm{ml}$. The data are replotted (Fig. 6) in terms of the $\mathrm{Rh}-\mathrm{ConA} /$ FITC-dextran ratio after correction for non-specific

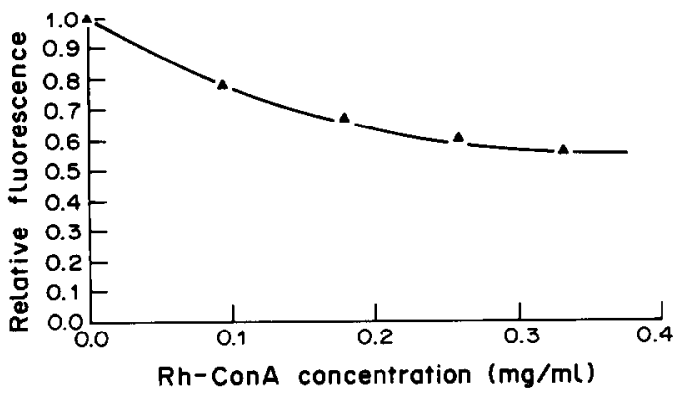

Fig. 5. Quenching effect of Rh-ConA on FITC fluorescence.

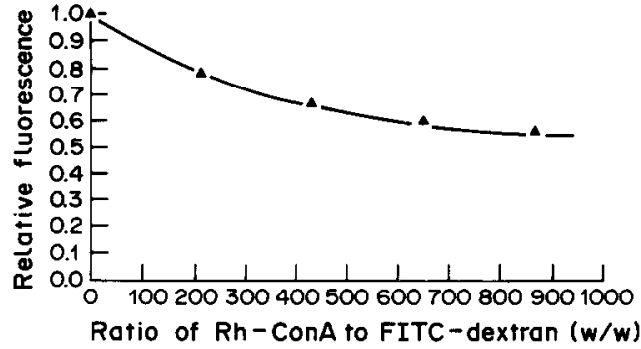

Fig. 6. Normalized quenching effect of $\mathrm{Rh}-\mathrm{ConA}$ on FITC fluorescence.

absorption of FITC fluorescence, i.e., the inner filter effect. It is easy to see from the figure that the relatively weak binding between ConA and dextran makes it important to have a large excess of $\mathrm{Rh}$-ConA in relation to FITC-dextran in order to achieve a significant amount of fluorescence quenching.

When glucose was titrated (reverse titration) by addition to a solution of $\mathrm{Rh}-\mathrm{ConA}$ and FITCdextran, there was the expected increase in fluorescence as the glucose displaced FITC-dextran from the ConA binding sites. Figure 7 shows the fraction of the theoretical maximum fluorescein signal recovered during glucose addition to two solutions with different $\mathbf{R h}-\mathrm{ConA} / \mathrm{FITC}$-dextran ratios. The theoretical maximum fluorescein signal was deduced from Fig. 6 for each Rh-ConA/FITC-dextran ratio by assuming that all the quenched signal would be restored when a large excess of glucose was added to the sample. For a weight ratio of 217 for $\mathrm{Rh}$-ConA/FITC-dextran, the fluorescence approached the theoretical maximum more rapidly than when the ratio was 870 . At a glucose concentration of $3.00 \mathrm{mg} / \mathrm{ml}, 60 \%$ of the signal was recovered with the 217 -ratio system, whereas only $\sim 50 \%$ was recovered with the 870-ratio system. As expected from the law of mass action, to achieve the largest signal response within a given glucose range, lower $\mathrm{Rh}$-ConA/FITC-dextran ratios should be used for low glucose concentrations and higher ratios for larger glucose concentrations. Although the relative signal change at low ratios is large for small changes

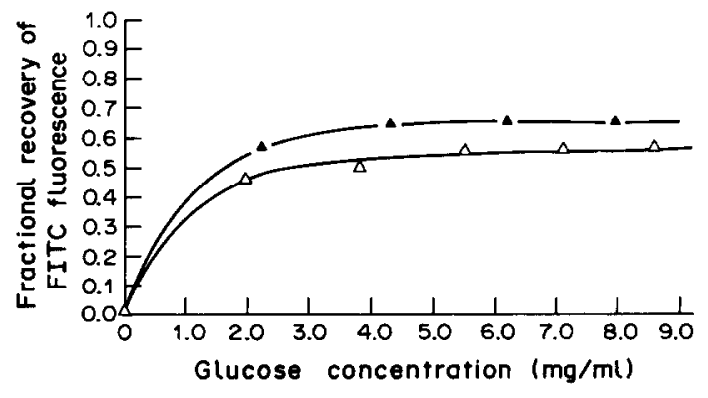

Fig. 7. Recovery of FITC fluorescence during glucose titration: $-\Delta-R h-C o n A / F I T C-d e x t r a n=217 \mathrm{w} / \mathrm{w}$; $-\triangle-\mathrm{Rh}-$ ConA/FITC-dextran $=870 \mathrm{w} / \mathrm{w}$. 
in glucose concentration, the absolute change is smaller. Therefore, both the detector sensitivity and the level of background fluorescence must be considered when specifying the $\mathrm{R} h-\mathrm{ConA} / \mathrm{FITC}$-dextran ratio.

\section{DISCUSSION}

The new optical homogeneous assay currently has a working range up to $2.00 \mathrm{mg} / \mathrm{ml}$ (Fig. 7). The linear region of the glucose titration curve can be arranged to cover a particular concentration range by adjusting the $\mathbf{R h}-$ ConA/FITC-dextran ratio. For a larger ratio, the titration curve will be linear to higher levels of glucose, but the sensitivity to small concentration changes will be diminished. There are several other ways of adjusting the sensitivity besides changing the reactant ratios. If the binding constant is increased, then better sensitivities are possible. The sensitivity can also be improved by adjusting the exciter/ quencher ratio, or by changing the fluorochrome pair so that the spectral overlap is larger, without adversely affecting the absorptivity. Finally, the size of the ligand analog can be decreased so that the fluorochromes are closer together on binding, which would permit more efficient energy transfer.

There is a practical lower limit to the assay, however. For measuring low glucose concentrations, the FITC-dextran concentration must also be low, making the fluorescein signal smaller and more prone to noise effects. Therefore, the FITC-dextran concentration must be large enough to ensure a good signal-to-noise ratio. We have typically used FITC-dextran concentrations of $0.5-5.0 \mu \mathrm{g} / \mathrm{ml}$ with very good results.

An upper limit to the dynamic range of the homogeneous glucose biosensor is set by the solubility of $\mathrm{Rh}-\mathrm{ConA}$. Because the sensor would initially be calibrated with all the components in solution, it is essential that the components are stable for the duration of the experiment. This means that the solution must not form insoluble aggregates, or the calibration would be changed. For the $\mathrm{Rh}-\mathrm{ConA} /$ FITC-dextran system, this is particularly critical because over a period of hours at $25^{\circ}$ ConA will become irreversibly aggregated and form precipitates. This process is even more rapid at $37^{\circ}$. We have performed several experiments to determine whether the stability of $\mathrm{Rh}-\mathrm{ConA}$ could be increased by physical means such as changes in temperature, ionic strength, $\mathrm{pH}$, or addition of synthetic polymers, but none of these methods was able to significantly increase the stability of ConA. We are currently trying several chemical modification methods, some of which have extended the stability of ConA to as long as two weeks at $37^{\circ}$ without adversely affecting the glucose binding. It is expected that there will be an optimum set of conditions for the modification procedure since there is a limited number of reaction sites on native ConA. The greater the number of reaction sites used during the chemical modification step, the fewer the number of remaining reaction sites available for Rhodamine labeling. We will be reporting on this work in a subsequent paper.

\section{CONCLUSIONS}

Our approach to the construction of fiber-optic biosensors based on competitive reactions for a bioreceptor between an analyte and a fluorescent analoganalyte has been extended by the use of homogeneous fluorescence quenching techniques. The approach described has several advantages over the heterogeneous systems previously reported.

1. It allows simultaneous monitoring of glucose concentrations from the FITC signal and continuous internal calibration from the Rhodamine signal to correct for light-source and detector sensitivity fluctuations.

2. The receptor does not have to be immobilized on the interior wall of the dialysis fiber, so the alignment between the optical and dialysis fibers is less critical.

3. Physical degradation of the dialysis fiber caused by the immobilization procedure is no longer important.

4. The assay can be easily scaled down from bench experiments to the sensor probe configuration because all the reaction components are in a single homogeneous solution.

5. The technique is general in nature, and any suitable Rhodamine-labeled bioreceptor, e.g., an antibody, $F_{A B}$ fragment, binding protein, can be added directly to the hollow fiber lumen along with the macromolecular ligand analog. This makes the sensor much more versatile for measuring hormones, metabolites 'and drugs, since there is no immobilization chemistry to be developed for each assay system.

On the other hand, in order to achieve the most efficient quenching, the overlap of the spectra for the two fluorophores needs to be optimized along with the relative concentrations of the two fluorophores. A higher quenching efficiency will increase the detection limit of the assay by increasing the signal-to-noise ratio. There are additional considerations that must be taken into account when designing a sensor, with regard to the relative concentrations and solubilities of the bioreceptor and the analyte-analog in the sensor compartment. The binding constant must be such that the assay gives a linear response over the desired analyte range when the bioreceptor and analyte analog are used at maximum solubility.

\section{REFERENCES}

1. M. Aizawa, Proc. Int. Mtg. Chem. Sensors, pp. 683-692. Elsevier, Amsterdam, 1983.

2. T. C. Pinkerton and B. L. Lawson, Clin. Chem., 1982, 28, 1946.

3. G. A. Rechnitz, Science, 1981, 214, 287. 
4. S. Suzuki, I. Satow and I. Karube, Appl. Biochem. Biotechnol., 1982, 7, 147.

5. J. Janata and R. J. Huber, Ion-Selective Electrode Rev., $1979,1,31$.

6. I. Lauks, SPIE Int. Soc. Optical Eng. Proc. Critical Reviews of Technology: Stratified Media, 1983, 387.

7. P. W. Cheung, W. J. Ko, D. J. Fung and S. H. Wong, Theory, Design and Biomedical Application of Solid State Chemical Sensors, P. W. Cheung, G. Fleming, W. H. Ko, M. R. Newman, (eds.), CRC Press, Cleveland, 1978.

8. P. Vadgama, J. Med. Eng. Technol., 1951 5, 293.

9. I. Chabay, Anal. Chem., 1982. 54, 1071A.
10. G. G. Vurek and R. L. Bowman, Anal. Biochem., 1969, 29, 238.

11. J. S. Schultz and G. Sims, Biotechnol. Bioeng. Symp., $1979,9,65$.

12. J. S. Schultz, S. Mansouri and I. J. Goldstein, Diabetes Care, 1982, 5, 245.

13. L. Stryer, Radiat Res. Suppl., 1960, 2, 432.

14. Idem, Ann. Rev. Biochem., 1978, 47, 819.

15. N. M. Green, Biochem. J., 1964, 90, 564.

16. E. F. Ullman, M. Schwarzenberg and K. E. Rubens, J. Biol. Chem., 1976, 251, 4172.

17. P. L. Khanna and E. F. Ullman, Anal. Biochem., 1980, 108, 156. 